\title{
Ultrathin Optics-Free Spectrometer with Monolithically Integrated LED Excitation
}

\author{
Tuba Sarwar and Pei-Cheng $\mathrm{Ku} * \mathbb{C}$
}

check for updates

Citation: Sarwar, T.; Ku, P.-C. Ultrathin Optics-Free Spectrometer with Monolithically Integrated LED Excitation. Micromachines 2022, 13, 382. https://doi.org/10.3390/ mi13030382

Academic Editors: Yi Zhang and Limin Xiao

Received: 31 January 2022

Accepted: 25 February 2022

Published: 27 February 2022

Publisher's Note: MDPI stays neutral with regard to jurisdictional claims in published maps and institutional affiliations.

Copyright: (C) 2022 by the authors. Licensee MDPI, Basel, Switzerland. This article is an open access article distributed under the terms and conditions of the Creative Commons Attribution (CC BY) license (https:// creativecommons.org/licenses/by/ $4.0 /)$.
Department of Electrical Engineering and Computer Science, University of Michigan, 1301 Beal Ave, Ann Arbor, MI 48109-2122, USA; tsarwar@umich.edu

* Correspondence: peicheng@umich.edu

\begin{abstract}
A semiconductor spectrometer chip with a monolithically integrated light-emitting diode was demonstrated. The spectrometer design was based on a computational reconstruction algorithm and a series of absorptive spectral filters directly built in to the photodetectors' active regions. The result is the elimination of the need to employ external optics to control the incident angle of light. In the demonstration, an array of gallium nitride $(\mathrm{GaN})$ based photodetectors with wavelength selectivity generated via the principle of local strain engineering were designed and fabricated. Additionally, a GaN based LED was monolithically integrated. An optical blocking structure was used to suppress the LED-photodetector interference and was shown to be essential for the spectroscopic functionality. A proof of concept using a reflection spectroscopy configuration was experimentally conducted to validate the feasibly of simultaneously operating the LED excitation light source and the photodetectors. Spectral reconstruction using a non-negative least squares (NNLS) algorithm enhanced with orthogonal matching pursuit was shown to reconstruct the signal from the reflection spectroscopy. Optics-free operation was also demonstrated.
\end{abstract}

Keywords: gallium nitride semiconductors; reflection spectroscopy; reconstructive spectrometers

\section{Introduction}

Optical spectroscopy is one of the most widely used characterization techniques in science and engineering. Miniaturizing the optical spectrometer can allow for a portable and handheld system and lead to new opportunities for Internet of Things (IoT) and labon-a-chip applications [1]. These applications can have important impacts in the fields of chemistry, biomedicine, food engineering, planetary exploration, point-of-care services, and the life sciences [2-11]. A spectrometer is a highly complex system consisting of optical, mechanical, and image processing units. Miniaturizing such a system is a nontrivial task involving the ability to integrate multiple material platforms and careful planning of performance tradeoffs, such as spectral resolution, sensitivity, system size, and cost. Among various approaches, spectrometers based on reconstructive algorithms [12] shift the complexity of processing spectral information from physical components to software computations [13-25]. With the steady growth of computational power per watt-dollar, this approach has become increasingly promising in constructing an extremely compact spectroscopic system.

A reconstructive spectrometer consists of a series of photodetectors with different spectral responses $R_{k}(\lambda)$, where $k$ denotes the $k$ th detector and $\lambda$ is the optical wavelength For an unknown spectrum $S(\lambda)$, the photocurrent generated by the $k$ th detector is given by $I_{k}=R_{k}(\lambda) S(\lambda)$. With a set of known $R_{k}(\lambda)$, one can reconstruct the unknown spectrum $S(\lambda)$ by inverting the above equation using a computational algorithm, e.g., the nonnegative least squares (NNLS) method. The spectral resolution is determined by the size of the $R_{k}(\lambda)$ matrix and how randomly each photodetector's spectral response relates to another. Ideally, there should be no correlations between any two photodetectors' responses 
and the number of photodetectors should be equal to the spectrometer's spectral range divided by the desired spectral resolution. For many applications, however, a reconstructive spectrometer can generate useful information even working as an underdetermined system. For example, the locations of a spectrum's peaks and the relative intensities between them are often sufficient information. Moreover, the reconstruction algorithms can be enhanced by adding constraints that can either be improved over time via learning or be constructed based on the specific characteristics of the application of interest [26-28]. This is highly desirable for many applications targeted by a miniaturized spectrometer.

A conventional semiconductor-based photodetector's spectral response cannot be easily tuned. Therefore, a reconstructive spectrometer has often been implemented with a series of filter/detector combinations. However, dispersive spectral filters are highly sensitive to the incident angle of light, requiring additional collimation optics which add to a system's bulkiness and weight. Recently, absorptive filters, either standalone or built into the photodetectors, have been successfully integrated into a reconstructive spectrometer $[13,14,18,21,29]$. Absorptive filters' spectral responses have a weak dependence on the incident angle of light [22]. As a result, collimation optics either become optional or can be replaced by other imaging optics to enable a spectrometer array for hyperspectral imaging.

Most spectroscopic applications require not only a detection system but also an excitation light source to provide a reference in reflection and transmission spectroscopy or to excite the analyte in fluorescence spectroscopy. Chip-scale integration of an LED (light-emitting diode) light source is an important part of miniaturizing a spectroscopic system. However, very few works have been carried out to address this need [5,30-33]. The main challenges include the heterogeneous integration of different material platforms for the light source and detectors, the already considerable size of the optical detection system preventing further integration of the light source, and interference between the light source and the spectral signal. In this work, we successfully demonstrated monolithic integration of LEDs and an array of wavelength-selective photodetectors. We experimentally validated the feasibility by showing the negligible interference of the on-chip LED due to the portion of light directly leaked to the detector array. We also showed an example of reflection spectroscopy using the on-chip LED and a notch filter to simulate an analyte absorbing at a specific wavelength.

\section{Materials and Methods}

The spectrometer design consists of an array of GaN-based wavelength-selective photodetectors [21]. When the strained InGaN quantum well active region is made into a nanopillar geometry, the strain relaxation shifts the emission wavelength of the quantum well to a short wavelength [34]. The strain relaxation is nonuniform and decreases from the edge to the center. As GaN exhibits a large piezoelectric polarization due to the in-plane strain, the strain relaxation leads to the change of the bandgap and the absorption cutoff wavelength. The amount of wavelength shift depends on the nanopillar's diameter and the emission wavelength of the original quantum well. In this work, the sample's epitaxial structure includes five periods of InGaN/GaN quantum wells sandwiched between a GaN pn junction. The room temperature (sample uncooled) electroluminescence peaked at $590 \mathrm{~nm}$ and can be tuned between 480 and $590 \mathrm{~nm}$ depending on the nanopillar diameter.

Figure 1a shows the device schematic. The device consists of an array of photodetectors, each constructed based on an array of GaN nanopillars comprising InGaN/GaN multiple quantum wells. It also has an LED which was made of GaN nanopillars. The nanopillar's diameter was varied to control emission and absorption properties. The sample was grown by metal-organic chemical vapor deposition (MOCVD) on a c-plane sapphire substrate. After growth, the sample was patterned using electron-beam lithography into a series of nanopillar arrays. Each array is either a photodetector or LED depending on the bias. Each array occupied a chip area of $100 \mu \mathrm{m}$ by $100 \mu \mathrm{m}$. All nanopillars in the same array have the same diameter. The spacing between two adjacent nanopillars was $250 \mathrm{~nm}$ for smaller nanopillar diameters and $2 \mu \mathrm{m}$ for larger ones. The nanopillars were 
formed using a two-stage etching process, first using inductively coupled plasma-reactive ion-etching (ICP-RIE) followed by an anisotropic wet etch in a $2 \%$ diluted $\mathrm{KOH}$ solution (AZ-400). Chromium was used as the etch mask. The wet etch step created a vertical sidewall, allowing the nanopillar diameter to be better controlled. The etching was stopped once we cleared the quantum well region. The resulting nanopillar height was $260 \mathrm{~nm}$. After etching, a conformal $50 \mathrm{~nm}$ thick $\mathrm{SiN}_{\mathrm{x}}$ layer was deposited to form electrical insulation around the nanopillars. The sample was then finished with planarization using $\mathrm{SiO}_{2}$ and electrode deposition ( $\mathrm{Ni}-\mathrm{Au}$ for $\mathrm{p}$-contact and $\mathrm{Ti}-\mathrm{Au}$ for $\mathrm{n}$-contact).

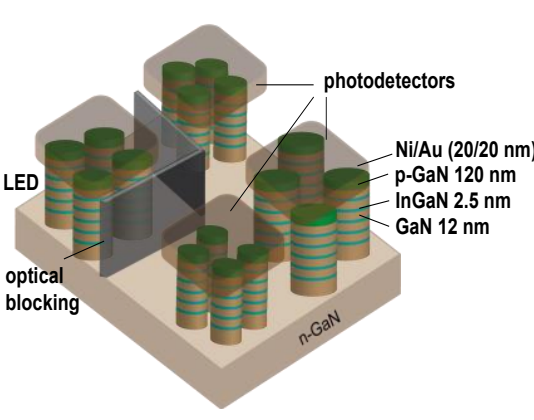

(a)

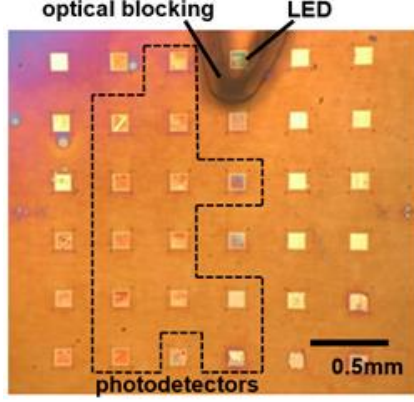

(b)

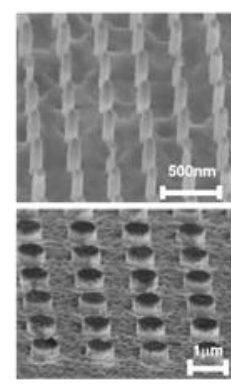

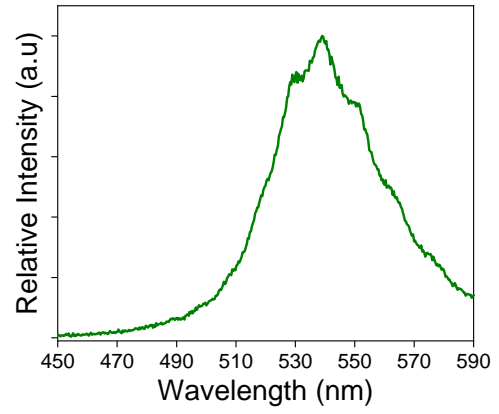

(c)

Figure 1. (a) Schematic of the proposed spectrometer chip with a monolithically integrated LED. As shown in the illustration, there are three photodetectors and one LED separated by an optical blocking structure. The three photodetectors exhibit different spectral responses due to the difference of the nanopillar diameter. (b) The optical image of the as-fabricated spectrometer chip with a monolithically integrated LED light source. Each yellow square is a nanopillar array with a chip area of $100 \mu \mathrm{m} \times 100 \mu \mathrm{m}$. The nanopillar diameters of the 13 arrays enclosed by the dashed line vary from $55 \mathrm{~nm}$ through thin film (see Section 3 for the diameter values). The black-colored structure is the enamel-coated PET optical blocking structure to suppress the direct capture of the LED emission by the photodetectors. The edge-to-edge spacing between two arrays is $0.3 \mathrm{~mm}$. The two scanning electron micrographs show two nanopillar arrays after etching but before planarization. The diameters of the nanopillars are $80 \mathrm{~nm}$ and $800 \mathrm{~nm}$ for the images on the top and bottom, respectively. (c) The emission spectrum of the on-chip LED (biased at 8V) measured by Ocean Optics HR2000. The LED device is also a nanopillar array with a diameter of $200 \mathrm{~nm}$.

Two sets, each containing a total of 14 nanopillar arrays were fabricated along with a few additional test structures. The optical image of the as-fabricated sample and the scanning electron micrographs of two of the arrays immediately after the patterning step are shown in Figure 1b. To choose the nanopillar diameters, we aim to distribute the absorption cutoff wavelengths of the nanopillar arrays evenly across a wide wavelength range. Previously, using a simple one-dimensional solid mechanics model which modeled the InGaN quantum well with a linear chain of atoms whose positions were determined from the balance of the strain relaxation due to the nanopillar geometry and the intrinsic compressive strain due to the lattice mismatch. The result is that the emission wavelength of each nanopillar array follows an exponential dependence $1-\sinh \kappa D / 2$ on the nanopillar's diameter $D$, where $\kappa$ describes the material's elastic property [34]. The emission wavelength of each nanopillar array was previously shown to be identical to the absorption cutoff wavelength at a zero bias [21]. To this end, we varied the nanopillar diameter from 55 to $100 \mathrm{~nm}$ with a $5 \mathrm{~nm}$ increment and from $100 \mathrm{~nm}$ to thin film with a larger increment to even out the emission wavelength distribution across the blue-green-orange range.

One of the 14 nanopillar arrays was chosen to operate in the LED mode with a forward bias applied. The emission spectrum was measured by Ocean Optics HR2000 and is shown in Figure 1c. As shown in Figure 1b, the LED is placed adjacent to the photodetector array. The spacing between each array is $0.3 \mathrm{~mm}$. Due to the proximity, the LED emission 
can be directly captured by the photodetectors. This interference can overwhelm the spectroscopic signal, which is likely to be much weaker than the emission along a direct path from the LED to the photodetectors. To address this challenge, we fabricated an optical blocking structure using polyethylene terephthalate (PET). We first coated the PET with black enamel. We then shaped the PET sheet into a wall-like structure and attached it to the sample surrounding the LED. Figure 2 shows that the PET optical blocking layer successfully suppressed the LED interference. The photocurrents due to the direct LED emission being captured by the photodetectors were significantly reduced to be within the same order of magnitude as the dark currents measured with the LED turned off.

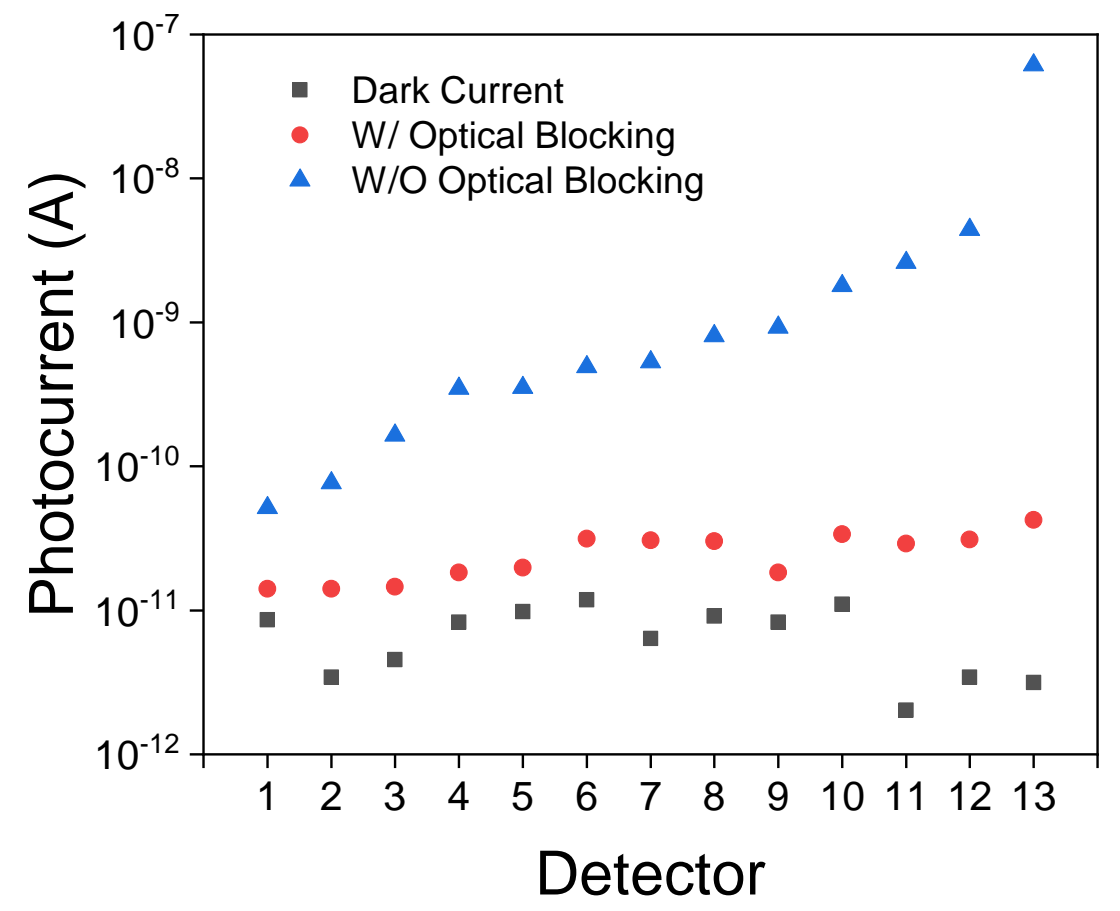

Figure 2. The photocurrents measured from the 13 photodetectors using a semiconductor parameter analyzer (Keithley 4200) under various scenarios: the dark currents measured when the on-chip LED was electrically disconnected; the photocurrents with and without the optical blocking structure integrated on the LED (shown in Figure 1a) which was biased at a constant 8V. LED emission reflected from an off-chip object is negligible. As a result, the photocurrents measured were the result of LED emissions being directly captured by the photodetectors along a direct path on the chip. With optical blocking, the photocurrent due to the LED interference can be considerably suppressed to be within the same order of magnitude as the dark current.

\section{Results}

Unlike a filter-based spectrometer, the reconstructive spectrometer's operating range is largely determined by the wavelength range in which the photodetectors' responses do not have a strong correlation. To determine the operating wavelength range of our device, we first measured each photodetector's responsivity between 450 and $590 \mathrm{~nm}$ with a $1 \mathrm{~nm}$ spectral resolution. All photodetectors were kept at a zero bias. The results are shown in Figure $3 \mathrm{a}, \mathrm{b}$. The responsivity shown uses a standard definition for a silicon photodetector and accounted for all the light not absorbed when passing between the nanopillars and $\sim 88 \%$ not absorbed when passing through the quantum wells. A stronger absorption, e.g., by increasing the nanopillar array's fill factor, can further enhance the responsivity. 


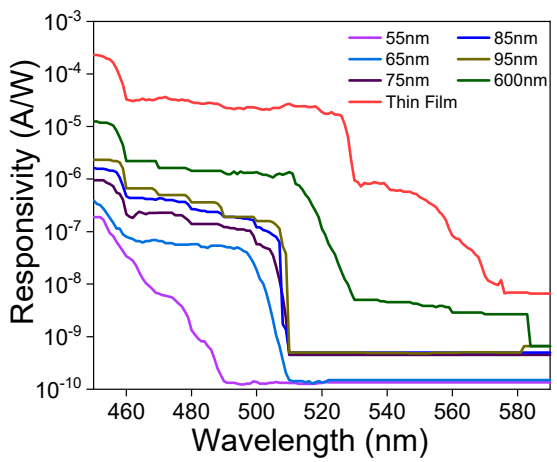

(a)

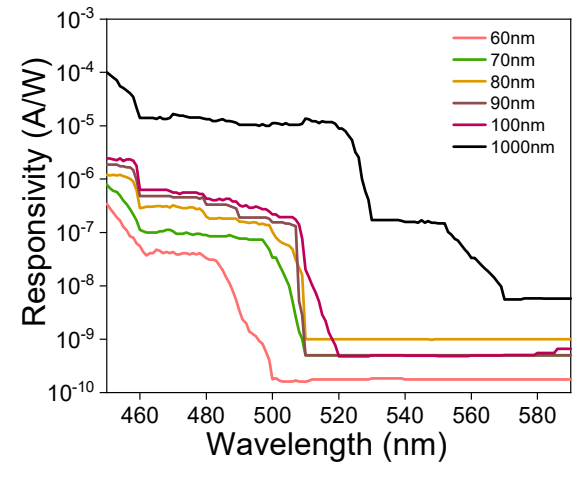

(b)

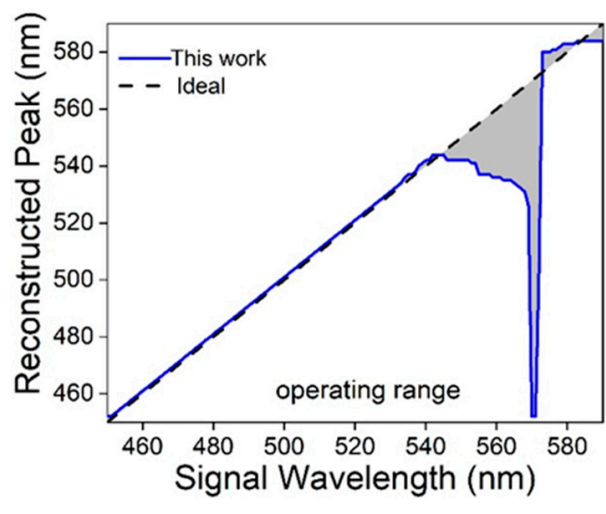

(c)

Figure 3. $(\mathbf{a}, \mathbf{b})$ The responsivities of the 13 photodetectors measured at a zero bias using a tunable monochromatic light source (adapted from [22]). The optical power at the sample was characterized separately using an integration sphere. The photocurrent was measured using a semiconductor parameter analyzer (Keithley 4200). The legend shows the nanopillar's diameter in each photodetector. (c) Determination of the spectrometer's operating range using an NNLS algorithm with a series of delta-function spectra between $450 \mathrm{~nm}$ and $590 \mathrm{~nm}$. The shaded area from 541 to $590 \mathrm{~nm}$ indicates the wavelength range in which the reconstruction has a large error. The result implies an operating range from $450 \mathrm{~nm}$ through $540 \mathrm{~nm}$.

Once the responsivities of all 13 photodetectors were obtained, we can determine the operating range of the spectrometer by performing the NNLS algorithm with a series of delta-function spectra, i.e., spectra with only a finite value at a specific wavelength and zeros everywhere else. The result is shown in Figure 3c. The reconstruction is accurate between 450 and $540 \mathrm{~nm}$. Although the emission wavelength of the photodetector ranges from 480 through $590 \mathrm{~nm}$, the operating window is not the same. The photodetectors' responses beyond $540 \mathrm{~nm}$ are not sufficiently random, which results in a large reconstruction error between 540 and $590 \mathrm{~nm}$. This can be remedied by increasing the responsivities of all photodetectors [22]. In contrast, although all photodetectors absorb strongly between 450 and $480 \mathrm{~nm}$, the correlation between their responsivities is weak enough to still lead to an accurate reconstruction.

Next, we demonstrated the feasibility of the monolithically integrated LED light source in the configuration of reflection spectroscopy. We placed a mirror approximately $4 \mathrm{~cm}$ from the sample, as shown in Figure 4a. We then inserted a notch filter (Thorlabs NF-533-17) with a center wavelength of $533 \mathrm{~nm}$ and a linewidth of $17 \mathrm{~nm}$ between the mirror and the sample. No other optics were used. In the experiment, the LED emission emanated from the sample passed through the notch filter, was reflected from the mirror, and passed through the filter another time before being absorbed by the photodetectors. The returned spectrum was measured by a commercial spectrometer and shown by the dashed curve in Figure $4 b$.

To reconstruct the reflected spectrum, we used an NNLS algorithm enhanced by the orthogonal matching pursuit (OMP) method to address a full range of spectral components simultaneously at multiple photodetectors [21]. We used a Gaussian basis with a $12 \mathrm{~nm}$ linewidth and a maximum iteration value of 141 . The reconstructed spectrum is shown in Figure $4 \mathrm{~b}$ with the blue solid curve which reasonably matches the spectrum measured by a commercial spectrometer (black dashed curve). The spectral reconstruction in this work is an underdetermined problem with only 13 photocurrent values available. As a result, only an approximate instead of an exact spectrum can be reconstructed. 


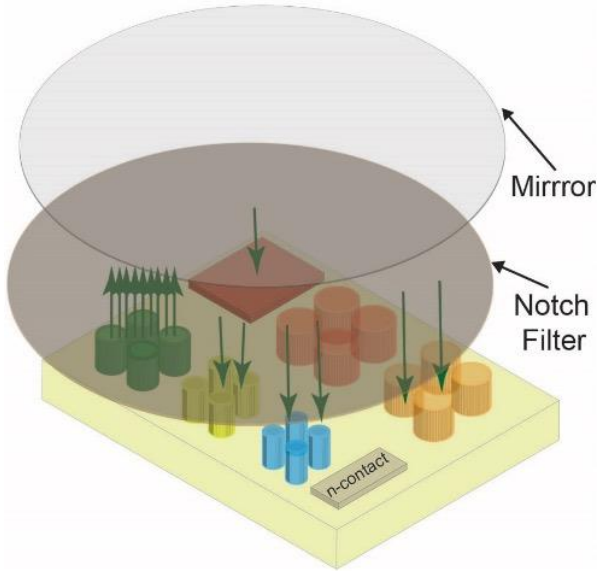

(a)

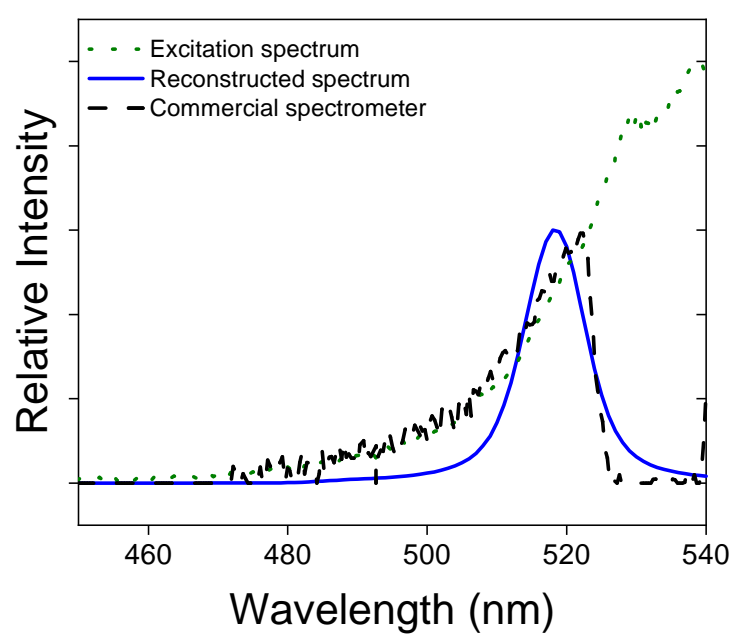

(b)

Figure 4. (a) The experimental configuration to demonstrate the feasibility of the monolithically integrated LED light source in reflection spectroscopy. A notch filter was used to modify the LED emission. Together with the mirror, the reflected spectrum simulated an analyte absorbing around $533 \mathrm{~nm}$ with an absorption linewidth of $17 \mathrm{~nm}$. The distance between the mirror and the chip is $4 \mathrm{~cm}$. No other optics other than the two shown were used. (b) The LED emission (green dotted curve), the reflected spectrum (black dashed curve) just above the chip captured by Ocean Optics HR2000, and the reconstructed spectrum (blue solid curve) using an OMP-enhanced NNLS algorithm.

\section{Discussion}

The reconstructive spectrometer chip designed, fabricated, and characterized in this work used a series of wavelength-selective photodetectors which exhibited a very weak dependence on the incident angle of light. As a result, collimation optics were not necessary for the functionality of the device. It was evident that the responsivities used in the spectral reconstruction in Figure $4 \mathrm{~b}$ were measured using a completely different illumination profile. The responsivities were measured using a monochromatic light focused onto the sample while the reflected spectrum in Figure 4 diverged significantly due to the incoherent nature of the LED emission. The results highlighted an important advantage of the reconstructive spectrometer using absorptive spectral filters, which in our case were built in as part of the photodetectors. The optionality of the collimation optics can enable a miniaturized spectrometer in an ultrathin form factor. One can also opt to integrate focusing lenses to increase the signal-to-noise level and/or to easily enable an array of spectrometers on the chip for hyperspectral imaging.

The spectroscopic operating range in this work can be expanded by reducing the correlation between the photodetectors' responses, e.g., by enhancing the absorption in the long-wavelength region. When some photodetectors' responsivities are very low, they effectively become non-participating and correlated, as 0 and 0 are always correlated. The enhanced absorption can allow more photodetectors to participate in the reconstruction algorithm. This was shown in [22].

The optical blocking in this work was fabricated using an enamel-coated PET sheet manually attached to the spectrometer chip. A more scalable approach can be to use a MEMS (microelectromechanical system) process. For example, when patterning the nanopillar arrays, one can keep a checkerboard pattern of GaN unetched. These walllike structures can then be heightened using PDMS (polydimethylsiloxane) which can be subsequently coated with a thin metal layer to be rendered light-blocking. As the LED is simply a forward-biased photodetector in our design, a photodetector array separated by optical blocking structures can enable a reconfigurable excitation spectrum for more versatile applications. 


\section{Conclusions}

In summary, we designed, fabricated, and demonstrated the feasibility of an optics-free spectrometer chip with a monolithically integrated LED light source. With 13 photodetectors, we successfully achieved spectroscopic functionalities in the wavelength range between $450 \mathrm{~nm}$ and $540 \mathrm{~nm}$. We also demonstrated the feasibility of reflection spectroscopy using the on-chip LED source and photodetectors simultaneously. The optical blocking structure successfully suppressed the direct capture of the LED emission by the photodetectors, which were as close as $0.3 \mathrm{~mm}$ from the LED. Finally, we showed that the spectrometer chip in this work did not require any external optics for its functionality. Therefore, the proposed spectrometer design can potentially enable a miniaturized spectroscopic system in an ultrathin film platform which can enable new opportunities, e.g., a wearable spectrometer chip to monitor physiological conditions in real time.

Author Contributions: Conceptualization, P.-C.K.; methodology, T.S. and P.-C.K.; experiment, T.S.; data analysis, T.S.; result interpretation, T.S. and P.-C.K.; writing-original draft preparation, T.S.; writing-review and editing, P.-C.K. All authors have read and agreed to the published version of the manuscript.

Funding: This research was funded by University of Michigan ESSI and the University of Michigan Blue Sky Initiative.

Data Availability Statement: The data presented in this study are available on request from the corresponding author.

Conflicts of Interest: The authors declare no conflict of interest.

\section{References}

1. Yang, Z.; Albrow-Owen, T.; Cai, W.; Hasan, T. Miniaturization of Optical Spectrometers. Science 2021, 371, eabe0722. [CrossRef] [PubMed]

2. Bacon, C.P.; Mattley, Y.; DeFrece, R. Miniature Spectroscopic Instrumentation: Applications to Biology and Chemistry. Rev. Sci. Instrum. 2004, 75, 1-16. [CrossRef]

3. Thenkabail, P.S.; Lyon, J.G. Hyperspectral Remote Sensing of Vegetation; CRC Press: Boca Raton, FL, USA, 2016 ; ISBN 9781439845387.

4. Chin, C.D.; Linder, V.; Sia, S.K. Lab-on-a-Chip Devices for Global Health: Past Studies and Future Opportunities. Lab A Chip 2007, 7, 41-57. [CrossRef] [PubMed]

5. Khan, Y.; Han, D.; Pierre, A.; Ting, J.; Wang, X.; Lochner, C.M.; Bovo, G.; Yaacobi-Gross, N.; Newsome, C.; Wilson, R.; et al. A Flexible Organic Reflectance Oximeter Array. Proc. Natl. Acad. Sci. USA 2018, 115, E11015-E11024. [CrossRef]

6. Wang, L.J.; Naudé, N.; Chang, Y.C.; Crivaro, A.; Kamoun, M.; Wang, P.; Li, L. An Ultra-Low-Cost Smartphone Octochannel Spectrometer for Mobile Health Diagnostics. J. Biophotonics 2018, 11, e201700382. [CrossRef] [PubMed]

7. Cai, F.; Lu, W.; Shi, W.; He, S. A Mobile Device-Based Imaging Spectrometer for Environmental Monitoring by Attaching a Lightweight Small Module to a Commercial Digital Camera. Sci. Rep. 2017, 7, 15602. [CrossRef] [PubMed]

8. Hardie, K.; Agne, S.; Kuntz, K.B.; Jennewein, T. Inexpensive LED-Based Optical Coating Sensor. IEEE Sens. J. 2017, 17, 6224-6231. [CrossRef]

9. De Lima, K.M.G. A Portable Photometer Based on LED for the Determination of Aromatic Hydrocarbons in Water. Microchem. J. 2012, 103, 62-67. [CrossRef]

10. Nugroho, F.A.A.; Darmadi, I.; Cusinato, L.; Susarrey-Arce, A.; Schreuders, H.; Bannenberg, L.J.; da Silva Fanta, A.B.; Kadkhodazadeh, S.; Wagner, J.B.; Antosiewicz, T.J.; et al. Metal-Polymer Hybrid Nanomaterials for Plasmonic Ultrafast Hydrogen Detection. Nat. Mater. 2019, 18, 489-495. [CrossRef] [PubMed]

11. OceanOptics Water Quality Monitoring: Chlorophyll a and Suspended Solids. 2015. Available online: https://oceanoptics.com/ wp-content/uploads/OceanView_Water-Quality-Monitoring.pdf (accessed on 17 January 2022).

12. Candès, E.J.; Romberg, J.; Tao, T. Robust Uncertainty Principles: Exact Signal Reconstruction from Highly Incomplete Frequency Information. IEEE Trans. Inf. Theory 2006, 52, 489-509. [CrossRef]

13. Bao, J.; Bawendi, M.G. A Colloidal Quantum Dot Spectrometer. Nature 2015, 523, 67-70. [CrossRef] [PubMed]

14. Guyot-Sionnest, P.; Ackerman, M.M.; Tang, X. Colloidal Quantum Dots for Infrared Detection beyond Silicon. J. Chem. Phys. 2019, 151, 060901. [CrossRef]

15. Wang, Z.; Yu, Z. Spectral Analysis Based on Compressive Sensing in Nanophotonic Structures. Opt. Express 2014, 22, 25608. [CrossRef] [PubMed]

16. Hu, X.; Liu, H.; Wang, X.; Zhang, X.; Shan, Z.; Zheng, W.; Li, H.; Wang, X.; Zhu, X.; Jiang, Y.; et al. Wavelength Selective Photodetectors Integrated on a Single Composition-Graded Semiconductor Nanowire. Adv. Opt. Mater. 2018, 6, 1800293. [CrossRef] 
17. Wang, Z.; Yi, S.; Chen, A.; Zhou, M.; Luk, T.S.; James, A.; Nogan, J.; Ross, W.; Joe, G.; Shahsafi, A.; et al. Single-Shot on-Chip Spectral Sensors Based on Photonic Crystal Slabs. Nat. Commun. 2019, 10, 1020. [CrossRef] [PubMed]

18. Yang, Z.; Albrow-Owen, T.; Cui, H.; Alexander-Webber, J.; Gu, F.; Wang, X.; Wu, T.C.; Zhuge, M.; Williams, C.; Wang, P.; et al. Single-Nanowire Spectrometers. Science 2019, 365, 1017-1020. [CrossRef] [PubMed]

19. Laux, E.; Genet, C.; Skauli, T.; Ebbesen, T.W. Plasmonic Photon Sorters for Spectral and Polarimetric Imaging. Nat. Photonics 2008, 2, 161-164. [CrossRef]

20. Park, H.; Dan, Y.; Seo, K.; Yu, Y.J.; Duane, P.K.; Wober, M.; Crozier, K.B. Filter-Free Image Sensor Pixels Comprising Silicon Nanowires with Selective Color Absorption. Nano Lett. 2014, 14, 1804-1809. [CrossRef]

21. Sarwar, T.; Cheekati, S.; Chung, K.; Ku, P.-C. On-Chip Optical Spectrometer Based on GaN Wavelength-Selective Nanostructural Absorbers. Appl. Phys. Lett. 2020, 116, 081103. [CrossRef]

22. Kim, J.; Cheekati, S.; Sarwar, T.; Ku, P. Designing an Ultrathin Film Spectrometer Based on III-Nitride Light-Absorbing Nanostructures. Micromachines 2021, 12, 760. [CrossRef] [PubMed]

23. Kim, C.; Lee, W.-B.; Lee, S.K.; Lee, Y.T.; Lee, H.-N. Fabrication of 2D Thin-Film Filter-Array for Compressive Sensing Spectroscopy. Opt. Lasers Eng. 2019, 115, 53-58. [CrossRef]

24. Kurokawa, U.; Choi, B.I.; Chang, C.C. Filter-Based Miniature Spectrometers: Spectrum Reconstruction Using Adaptive Regularization. IEEE Sens. J. 2011, 11, 1556-1563. [CrossRef]

25. Brown, C.; Goncharov, A.; Ballard, Z.S.; Fordham, M.; Clemens, A.; Qiu, Y.; Rivenson, Y.; Ozcan, A. Neural Network-Based On-Chip Spectroscopy Using a Scalable Plasmonic Encoder. ACS Nano 2021, 15, 6305-6315. [CrossRef] [PubMed]

26. Zhang, S.; Dong, Y.; Fu, H.; Huang, S.L.; Zhang, L. A Spectral Reconstruction Algorithm of Miniature Spectrometer Based on Sparse Optimization and Dictionary Learning. Sensors 2018, 18, 644. [CrossRef] [PubMed]

27. Meng, J.; Cadusch, J.J.; Crozier, K.B. Plasmonic Mid-Infrared Filter Array-Detector Array Chemical Classifier Based on Machine Learning. ACS Photonics 2021, 8, 648-657. [CrossRef]

28. Oliver, J.; Lee, W.; Park, S.; Lee, H.-N. Improving Resolution of Miniature Spectrometers by Exploiting Sparse Nature of Signals. Opt. Express 2012, 20, 2613. [CrossRef] [PubMed]

29. Park, H.; Crozier, K.B. Vertically Stacked Photodetector Devices Containing Silicon Nanowires with Engineered Absorption Spectra. ACS Photonics 2015, 2, 544-549. [CrossRef]

30. Rae, B.R.; Griffin, C.; McKendry, J.; Girkin, J.M.; Zhang, H.X.; Gu, E.; Renshaw, D.; Charbon, E.; Dawson, M.D.; Henderson, R.K. CMOS Driven Micro-Pixel LEDs Integrated with Single Photon Avalanche Diodes for Time Resolved Fluorescence Measurements. J. Phys. D Appl. Phys. 2008, 41, 094011. [CrossRef]

31. Huang, C.; Zhang, H.; Sun, H. Ultraviolet Optoelectronic Devices Based on AlGaN-SiC Platform: Towards Monolithic Photonics Integration System. Nano Energy 2020, 77, 105149. [CrossRef]

32. Zhang, H.; Huang, C.; Song, K.; Yu, H.; Xing, C.; Wang, D.; Liu, Z.; Sun, H. Compositionally Graded III-Nitride Alloys: Building Blocks for Efficient Ultraviolet Optoelectronics and Power Electronics. Rep. Prog. Phys. 2021, 84, 044401. [CrossRef]

33. Lyu, Q.; Jiang, H.; Lau, K.M. Monolithic Integration of Ultraviolet Light Emitting Diodes and Photodetectors on a P$\mathrm{GaN} / \mathrm{AlGaN} / \mathrm{GaN} / \mathrm{Si}$ Platform. Opt. Express 2021, 29, 8358. [CrossRef] [PubMed]

34. Teng, C.-H.; Zhang, L.; Deng, H.; Ku, P.-C. Strain-Induced Red-Green-Blue Wavelength Tuning in InGaN Quantum Wells. Appl. Phys. Lett. 2016, 108, 071104. [CrossRef] 